\title{
Eco-Efficient Packaging Material Selection for Fresh Produce: Industrial Session
}

\author{
Nouredine Tamani ${ }^{1}$, Patricio Mosse ${ }^{2}$, Madalina Croitoru ${ }^{1}$, Patrice Buche ${ }^{2}$, \\ Valérie Guillard ${ }^{2}$, Carole Guillaume ${ }^{2}$, Nathalie Gontard ${ }^{2}$ \\ ${ }^{1}$ LIRMM, University Montpellier 2, France \\ ${ }^{2}$ UMR IATE INRA 2 place Pierre Viala Montpellier, France
}

\begin{abstract}
Within the framework of the European project EcoBioCap (ECOefficient BIOdegradable Composite Advanced Packaging), we model a real world use case aiming at conceiving the next generation of food packagings. The objective is to select packaging materials according to possibly conflicting requirements expressed by the involved parties (food and packaging industries, health authorities, consumers, waste management authority, etc.). The requirements and user preferences are modeled by several ontological rules provided by the stakeholders expressing their viewpoints and expertise. To deal with these several aspects $\left(\mathrm{CO}_{2}\right.$ and $\mathrm{O}_{2}$ permeance, interaction with the product, sanitary, cost, end of life, etc.) for packaging selection, an argumentation process has been introduced.
\end{abstract}

\section{Introduction}

Within the framework of the European project EcoBioCap (www.ecobiocap.eu) about the design of next generation packagings using advanced composite structures based on constituents derived from the food industry, we aim at developing a Decision Support System (DSS) to help parties involved in the packaging design to make rational decisions based on knowledge expressed by the experts of the domain.

The DSS is made of two parts, as depicted in Figure 1:

1. a flexible querying process which is based on a bipolar approach dealing with imprecise data [8] corresponding to the characteristics related to the food product to pack like the optimal permeance, the dimension of the packaging, its shape, etc.,

2. an argumentation process which aims at aggregating several stakeholders (researchers, consumers, food industry, packaging industry, waste management policy, etc.) requirements expressed as simple textual arguments, to enrich the querying process by stakeholders' justified preferences. Each argument supports/opposes a choice justified by the fact that it either meets or not a requirement according to a particular aspect of the packagings.

We implementated of the second part of the DSS, called argumentation system, which aims at aggregating preferences associated with justifications expressed by stakeholders about the characteristics of a packaging. This module 


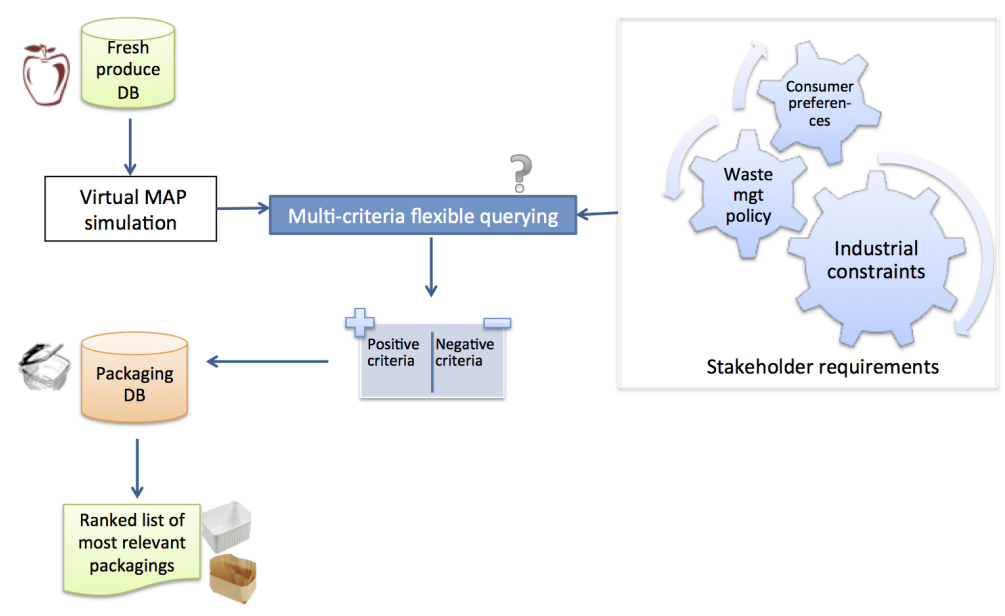

Fig. 1. Global insight of the EcoBioCap DSS.

has as inputs stakeholders' arguments supporting or opposing a packaging choice which could be seen as preferences combined with their justifications, and returns consensual preferences which may be candidates to enrich the bipolar querying system.

The DSS consists of two steps: (i) aggregating possibly conflicting needs expressed by the involved several parties (ii) querying a database of packagings with the resulting aggregation obtained at point (i).

In this real case, packagings have to be selected according to several aspects or criteria (permeance, interaction with the packed food, end of life, etc.), highlighted by the expressed stakeholders' arguments. The problem at hand does not simply consist in addressing a multi-criteria optimization problem [4]: the domain experts would need to be able to justify why a certain packaging (or set of possible packagings) are chosen. Argumentation theory in general $[9,3,11]$ is actively pursued in the literature, some approaches even combining argumentation and multi criteria decision making [2].

\section{Approach}

Stakeholder's set of arguments $i$ is then modeled as concepts, facts and rules to build a partial knowledge bases $\mathcal{K}_{\mathcal{I}_{i}}$. The union of every stakeholder knowledge base $\mathcal{K}=\bigcup_{i=1, \ldots, n} \mathcal{K}_{\mathcal{I}_{i}}$ will be used to instantiate the ASPIC [1] argumentation system, as shown on Figure 2.

The main salient points of our work in the EcoBioCap project are the following:

1. A DLR-Lite $[7,5]$ ontology extended to a negation to express stakeholders' arguments about packaging characteristics as combination of concepts 


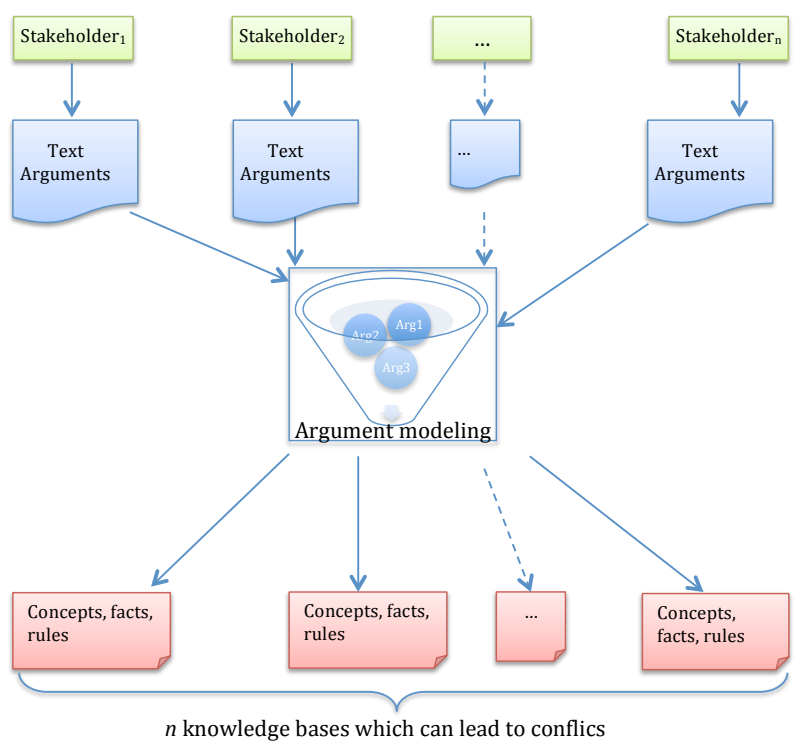

Fig. 2. The global knowledge base of the system.

(defined as $m$-ary relations connected to a database) and inference rules (specified as subsumptions). The language is detailed in the technical report [12],

2. An instantiation of ASPIC argumentation system $(A S)$ with the proposed DLR-Lite logical language. The instantiated ASPIC $A S$ satisfies the rationality postulates [6], please see details in [12],

3. The study of the influence of the modeling rules on the argumentation results. We showed the limitation of the crisp split of the inference rules into defeasible and strict, and we propose to overcome this limitation a viewpoint approach in which arguments are gathered according to packaging aspects. Each viewpoint delivers subsets of non-conflicting arguments supporting or opposing a kind of packaging according to a single aspect (shelf life parameters, cost, materials, sanitary, end elf life, etc.),

4. The use of the argumentation results for a bipolar querying of the packaging database. Indeed, we can gather the results onto positive and negative collections. We can then deduce automatically such queries from the collections the users formed during the argumentation process.

5. Implementation of the approach. A java GXT/GWT web interface was developed and a open version is accessible on http://pfl.grignon.inra.fr/EcoBioCapProduction/. 


\section{Architecture of the argumentation system}

As illustrated in Figure 3, the proposed argumentation system relies on 5 main modules, described below.

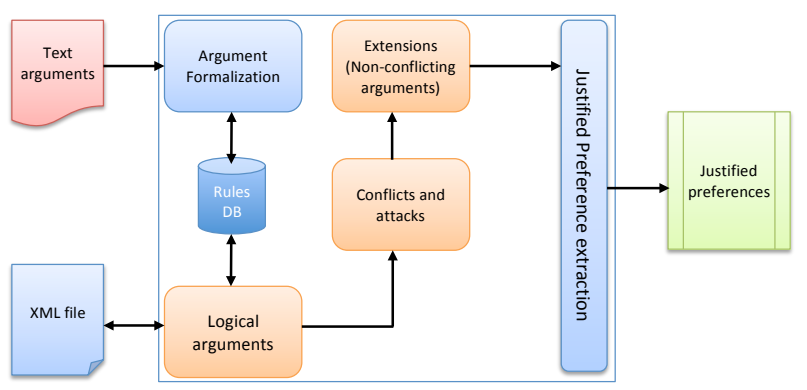

Fig. 3. The architecture of the argumentation system.

- Argument formalization module: this module implements a user-friendly interface for a semi-automatic translation of text arguments into a formal representation made of concepts and rules (claims and hypothesis). A graphical representation of the expressed rules is also built as the users formalize their text arguments. The formal representation obtained is finally saved in a database for a persistent storage allowing to reload argumentation projects without rebuilding all the arguments and to reuse also the already formatted rules in other projects.

- Logical arguments: this module receives as inputs the list of concepts and rules corresponding to text arguments. This list can be the result of the formalization module or given by the user as an XML file. Then, by a derivation process, this module builds all possible arguments according to the logical process defined in ASPIC/ASPIC+ logic-based argumentation frameworks $[1,10]$ and reused in $[13,14]$. This modules implements also a function to export the argument list into an XML document.

- Conflicts and attacks: this module relies on the logical arguments built by the previous module. According to the negation operator, it detects all the conflicts among arguments and models them as attacks with respect to the definition of attacks introduced in [13,14]. The output of this module is an argumentation graph made of arguments (nodes) and attacks (edges).

- Extensions: an extension is a subset of non-conflicting (consistent) arguments which defend themselves from attacking arguments. The computation of extensions is made under one semantics (preferred, stable, grounded, etc.) as defined in [9]. This module allows the computation of one or all semantics considered (preferred, stable, grounded, eager, semi-stable). We notice that 
theoretically we can get empty extensions under any semantics. This situation occurs when a user expresses at least one self-defeated argument, which is not attacked by any other argument, but attacks all the others. This kind of arguments are called contaminating arguments [15]. The current version of the system detects the rules leading to such arguments and discards them before performing the process of extension computations.

- Extraction of the justified preferences: the computation of extensions delivers one or several extensions. In the case of several extensions, the system lets the users selecting the more suitable one according to their objectives. The selected extension is then used to extract corresponding preferences underlying the contained concepts. These preferences are expressed as a list of couples (attribut, value), where attribute stands for a packaging attribute as defined in the packaging database schema of the flexible querying system part of the DSS, and value is the preferred value expressed for the considered attribute.

\section{Conclusion}

We applied an argumentation approach on a real use case from the industry allowing stakeholders to express their preferences and providing the system with stable concepts and subsumptions of a domain. We have proposed an argumentation system in which each criterion (attribute or aspect) is considered as a viewpoint in which stakeholders express their arguments in homogenous way. Each viewpoint delivers extensions supporting or opposing certain choices according one packaging aspect, which are then used in the querying process. The approach is implemented as freely accessible web application.

\section{References}

1. L. Amgoud, L. Bodenstaff, M. Caminada, P. McBurney, S. Parsons, H. Prakken, J. Veenen, and G. Vreeswijk. Final review and report on formal argumentation system.deliverable d2.6 aspic. Technical report, 2006.

2. L. Amgoud and H. Prade. Using arguments for making and explaining decisions. Artificial Intelligence, 173(3-4):413-436, 2009.

3. P. Besnard and A. Hunter. Elements of Argumentation. The MIT Press, 2008.

4. D. Bouyssou, D. Dubois, M. Pirlot, and H. Prade. Decision-making process Concepts and Methods. Wiley, 2009.

5. D. Calvanese, G. D. Giacomo, D. Lembo, M. Lenzerini, and R. Rosati. Data complexity of query answering in description logics. In $K R$, pages 260-270, 2006.

6. M. Caminada and L. Amgoud. On the evaluation of argumentation formalisms. Artificial Intelligence, 171:286-310, 2007.

7. S. Colucci, T. D. Noia, A. Ragone, M. Ruta, U. Straccia, and E. Tinelli. Semantic Web Information Management, chapter 19 : Informative Top-k retrieval for advanced skill management, pages 449-476. R. De Virgilio et al., springer-verlag belin heidelberg edition, 2010. 
8. S. Destercke, P. Buche, and V. Guillard. A flexible bipolar querying approach with imprecise data and guaranteed results. Fuzzy sets and Systems, 169:51-64, 2011.

9. P. M. Dung. On the acceptability of arguments and its fundamental role in nonmonotonic reasoning, logic programming and n-persons games. Artificial Intelligence, $77(2): 321-357,1995$.

10. H. Prakken. An abstract framework for argumentation with structured arguments. Technical report, Department of Information and Computing Sciences. Utrecht University., 2009.

11. I. Rahwan and G. Simari. Argumentation in Artificial Intelligence. Springer, 2009.

12. N. Tamani and M. Croitoru. Fuzzy argumentation system for decision making. https://drive.google.com/file/d/0B0DPgJDRNwbLdE5wdzFQekJocXM/edit?usp=sharing. Technical report, INRIA LIRMM, 2013.

13. N. Tamani, M. Croitoru, and P. Buche. A viewpoint approach to structured argumentation. In M. Bramer and M. Petridis, editors, The Thirty-third SGAI International Conference on Innovative Techniques and Applications of Artificial Intelligence, pages 265-271, 2013.

14. N. Tamani, M. Croitoru, and P. Buche. Conflicting viewpoint relational database querying: an argumentation approach. In L. Scerri and B. Huhns, editors, Proceedings of the 13th International Conference on Autonomous Agents and Multiagent Systems (AAMAS 2014),, 2014. to appear.

15. Y. Wu. Between argument and conclusion. Argument-based approaches to discussion. Inference and Uncertainty. PhD thesis, Université du Luxembourg, 2012. 